\title{
Selection and Ranking of Fog Computing-Based IoT for Monitoring of Health Using the Analytic Network Approach
}

\author{
Dong Xue, ${ }^{1}$ Shah Nazir ${ }^{(D)},{ }^{2}$ Zhiqiang Peng $\mathbb{D}^{1},{ }^{1}$ and Hizbullah Khattak ${ }^{3}{ }^{3}$ \\ ${ }^{1}$ Sichuan Tourism University, Chengdu 610100, China \\ ${ }^{2}$ Department of Computer Science, University of Swabi, Swabi, Khyber Pakhtunkhwa, Pakistan \\ ${ }^{3}$ Department of Information Technology, Hazara University Mansehra, Mansehra, Khyber Pakhtunkhwa, Pakistan \\ Correspondence should be addressed to Shah Nazir; snshahnzr@gmail.com and Zhiqiang Peng; peszq@sina.com
}

Received 24 March 2021; Accepted 31 July 2021; Published 9 August 2021

Academic Editor: Muhammad Ahmad

Copyright (c) 2021 Dong Xue et al. This is an open access article distributed under the Creative Commons Attribution License, which permits unrestricted use, distribution, and reproduction in any medium, provided the original work is properly cited.

\begin{abstract}
Numerous raised areas are established in the field of fog computing (FC), applied for various purposes, and are evaluated for running analytics on various devices including devices of internet of things and many others in a disseminated way. FC progresses the prototype of cloud computing to network edge leading various possibilities and services. FC improves processing, decision, and intervention to take place through devices of IoT and communicate essential details. The idea of FC in healthcare based on frameworks of IoT is exploited by determining dispersed delegate layer of comprehension between the cloud and sensor hubs. The clouds suggested systems improved to overcome several challenges in ubiquitous frameworks of medical services such as energy efficiency, portability, adaptableness, and quality issues by accommodating right to take care of definite weights of the distant medical services group and sensor networks. The proposed research work has considered the analytic network process (ANP) for selection and ranking of FC-based IoT for health monitoring systems. The approach works in situation when complexity arises for health monitoring. Results of the study show the success of the research for facilitating healthcare.
\end{abstract}

\section{Introduction}

Fog computing is a structure positioned anywhere between the cloud and the data source where information is computed and stored and the applications are placed to operate the requirements for some specific purpose. FC, like edge computing, takes the assistance of cloud's and power nearby to where information is formed and functioned. Fog computing and edge computing are used by various people as both involve taking understanding and computation together for the formation of information. This is generally performed for reliability improvement but can also be performed for other reason such as adherence and protection. Abundant platforms are recognized in the field of FC which are applied for various purposes and are assessed for successive analytics on different devices comprising IoT devices and several others in a disseminated means. FC improves the prototype of cloud computing to the network edge, leading various potentials and services. FC improves processing, decision, and intervention to take place through devices of IoT and spread essential details.

FC plays an important role in healthcare, and with the help of this platform, various mechanisms are proposed. Patients suffering from chronic disease are monitored through fog computing [1]. The challenging task is the collection of context-sensitive data associated with patient health. The use of sensor-to-cloud is not only feasible, and the layer of fog computing can make a difference. By doing this, the efficiency of the entire system grows. The issues of security and deployment of fog computing layer are also analyzed. The large growth and developments in the IoT have offered excessive possibilities in the sector of healthcare. The fitness trackers, wireless technology, and body sensors have significant effect on health system reliability and living efficiency. The applications of wearable devices are increasing which measure physiological factors, enhance adherence to exercise in various populations from athletes to patients, and promote health. Fog-assisted computational 
efficient wearable sensor networks have been considered for monitoring health through IoT. The applications of wearable devices for monitoring respiratory frequency, hear rate, and movement cadence through physical activity are examined. The collected data through the sensor are uploaded to the connection of IoT system Ethernet module, and the official individual access is provided to monitor the health of athletics through Internet. The wearable devices and its applications demonstrate how the resources of computing cost can reduce while maintaining health requests to access information of health stored in cloud and fog distributed setting. The effectiveness, reliability, and user-friendliness are demonstrated through results of the experiments [2].

Health clinics for sports performance services and medicine of sports team are presented with the applications of technology for supporting athletes return to play in various areas of sports. For analyzing and monitoring health operative optimization of sports person, machine learning algorithms are presented. With the help of experimental results and discussions, the efficiency of the designed system is assessed [3]. Outside the environment of hospital, wireless body sensor nodes are used to monitor patient based on realtime IoT. These devices include bio-sensor for capturing signals from body of patient and transmitting the collected signals through wireless transmitters to a server in real time. The study presented a real-time scheme of encoding performing approximation of wavelet coefficients for sparse encoding of bio-signals and iterative thresholding for reducing bandwidth consumption and reducing energy of the wireless body sensor node. The proposed system results presented energy improvement of system level $96 \%$ with an impact of $2 \%$ on signal quality [4]. The research has demonstrated a novel "Intelligent" skin monitoring device allowing patients of rural areas to remotely monitor diseases of skin. The approach includes cloud-based IoT and artificial intelligence for analyzing medical images and predictions of disease. The research also addresses the impact of the season. The proposed framework offers diagnosis and prevention for addressing the issues faced by people of remote areas with less facility of skincare [5].

The research work presented in this paper has considered and used the analytic network process approach for selection and ranking of FC based IoT for monitoring of health. The approach works in situation when complexity arises for health monitoring.

The paper is organized as follows. Section 2 depicts the literature of the proposed research inline with FC-based IoT for healthcare. Section 3 represents the literature for evaluation and quality assurance of FC-based IoT for monitoring of health. Selection and ranking of FC-based IoT for health monitoring are briefly given in Section 4 . Section 5 shows the conclusion of the paper.

\section{Related Work}

Various approaches are presented for monitoring healthrelated activities. Various generic monitoring systems are existing. Dhingra et al. [6] have offered research on the analysis integrated fog and distributed computing arrangement acquainted with limitations of latency, realtime analytics, and network congestion of basic cloud services for monitoring of traffic. The planned approach of cloud and fog framework is linked with Twitter for sending alerts concerning congestion of traffic. The system of IoT and fog-based healthcare for identification and regulation of Chikungunya virus (CHV) is introduced. Fuzzy-C mean clustering is used to evaluate the infected users and to provide customers from the fog layer with thoughtful and alert concerns quickly. In addition, social network analysis is used on cloud workers to speak to the CHV outbreak. With the help of the social network analysis graph, the outbreak role index is measured which signifies the possibility of any user to spread or receive the infection. It produces alerts of warning to healthcare agencies and government for controlling $\mathrm{CHV}$ outbreak in risk proven regions. The advantages of the research concerning cloud and fog services for obtaining efficient bandwidth with good quality of service are shown by the experimental results [7].

To find, shape, and screen customers affected with mosquito-borne diseases (MBDs), a new system of IoT sensors, fog computing, and cloud computing is planned to classify, differentiate, and monitor the users with MBD infection. The research aims to stop the outbreak of MBDs at the initial stage. The system is proposed, the similarity factor is measured for differentiating MBDs, and J48 was considered for classification of category for each user infection. The preventive procedures are formed in a split second and sent to the client from the fog layer if any anomaly should occur. Radio frequency identification is considered for identification of the closeness of customers. Temporal network analysis uses proximity information to monitor and talk to the present state of the flare-up of the MBDs. In order to find MBDs, the examining assessments of the system resulted low error rate and high accuracy of 94\% [8]. A comprehensive set of topology control (TC) approach for managing and constructing a large-scale smart city network of IoT is presented [9]. The problem of TC is approached in two phases consisting of construction and maintenance phase. In the first phase, a cost effective network of IoT including fog gateways is built while in the second phase the resource utilization is optimized. For realizing the objectives, efficient algorithms are proposed and wide-ranging simulation is done based on experimental and real IoT data sets. The effectiveness of the approach is demonstrated and compared with the available algorithms. The efficient development of innovation in medical care makes it emphatically possible for clinicians to screen and go to patients with constant illness to provide patients with adequate treatment in remote areas.

A scientific examination of robotics telesurgery established on 5G, artificial intelligence, and tactile internet is presented. The paper has described the issues and challenges faced in telesurgery. A fog-based supporting interactive model is demonstrated for expediting and reducing the process latency [10]. The influence of fog computing paradigm in healthcare is studied highlighting the key assistances concerning latency, power consumption, and network usage. With foundation of these parameters, the 
fog-based supporting health monitoring system is planned and its evaluation performance is carried out. The experimental results of the approach demonstrated the enhancement possibilities for minimizing data traffic with security enhancement on health information and providing optimized insights of patient health [11].

\section{Evaluating and Quality Assurance of FC- Based IoT for Observing Health}

In the literature so far, various approaches and mechanisms are available to monitor healthcare activities. Verma et al. [12] have presented a new IoT-fog cloud supporting physical system to diagnose and stage classification of ulcerative colitis (UC) through Naïve Bayes classifier and deep neural network. Real-time alert generation is the key point of the study from fog layer in the situation of emergency needed to the user with UC. Haghi Kashani et al. [13] analyzed that the quality of service confirming approaches comes under three categories including service management, application management, and communication management for the years during 2013 till October 2018. The planned study has demonstrated the tools, merits, demerits, evaluation types, and factors of quality of service. Lastly, the challenges and issues based on the reviewed studies have been suggested which need further research for the approaches in fog computing. A business process model and notation extension for enabling the IoT aware business process modeling are proposed at first. The approach considers the heterogeneous IoT and non IoT resources, quality of service constraints, resource capacities, and so forth. Secondly, the approach has considered a novel IoT-fog cloud-based architecture. Thirdly, the research has modeled the proposed BPMN 2.0 extension, smart autistic child, and coronavirus disease 2019 monitoring system. The effectiveness of the proposed system is illustrated with the help of extensive experiments [14]. Figure 1 graphically decipts the IoT-based health monitoring system [4]. The figure consists of three tiers and the details. This figure mainly discusses the IoTbased health monitoring system.

Figure 2 represents the components involved and architecture [15]. The figure contains IoT sensors, GPS module, WiFi module, collected health data, wireless local gateway, wireless remote gateway, and controller.

Karatas and Korpeoglu [16] have considered fog computing and geographically distributed cloud-based architecture of IoT and planned an approach to place IoT data into the components such as fog and cloud data centers. Data are stored in various types, and each of the data type is required by several applications. The research has modeled the problem of data placement and proposed the effective and efficient algorithm for placement of data produced and disbursed through location effectively accessed by applications which require the type of data. For evaluating the proposed research, extensive experiments are done and the effectiveness of the research is shown.

Various searching processes were done in order to obtain associated materials. The Springer library was searched for the query defined for the search process. The materials were graphically shown for understanding. Figure 3 graphically represents the details of the papers searched in the given library.

The disciplines of the papers were also studied which are shown in Figure 4.

Alemneh et al. [17] have proposed a two-way subjective logic-based trust management enabling a service requester for verifying a service provider is offering a secure and reliable service and the service provider can check the trustworthiness of the service requester. Aslam et al. [18] have presented a solution of an automated fog node audit certification approach which certifies the secure fog layer by the proposed fog layer promising mechanism. Figure 5 shows the details of the papers searched in the IEEE library. The figure shows that more papers are published as conference papers.

Figure 6 is to represent the locations of the conferences where the papers are published.

Figure 7 depicts the topics of publications.

The details of publication titles and publications are shown in Figure 8.

Bandopadhaya et al. [19] have planned an integrated solution of healthcare monitoring for the soldiers implemented in adversative surroundings circumstances through the IoT with distributed computing. Each health parameters of individual requires to be monitored on a real-time basis and succeeding examination of the dataset for commencing suitable healthcare support on time. Bharathi et al. [20] have presented an energy efficient particle swarm optimizationbased clustering approach for efficient selection of cluster head amongst different devices of IoT. The devices of IoT for healthcare sensing data are categorized into a form of cluster, and a cluster head will be elected by the use of the approach. The data are forwarded by the cluster head to the cloud server. Fog devices are used for transmitting data of the IoT devices to the cloud server. The ANN algorithm is used as the classification model for diagnosis of the healthcare data for identification of the severity of disease. Debauche et al. [21] presented the health monitoring system based on a fog IoT cloud through environmental signal and physiological permitting to generate relative information for the daily activities. The proposed system monitors behavioral changes and health state of elderly people. Recovery and monitoring rehabilitation processes of patients are provided by the proposed system. The system consists of local gateway for storing data locally, wireless sensor network, and Lambda cloud architecture for processing data.

The ScienceDirect library was considered as search library for achieving associated materials. Figure 9 shows the details of the types of articles along with number of articles.

In this library, the year wise search process was also done to show the increase/decrease in research in the area. Figure 10 depicts the year wise details of paper. The figure shows that there is increase in research work in the area.

The publication titles were searched in the same library, and it is shown in Figure 11.

The subject areas along with publications were found in the same library. Figure 12 graphically represents the subject areas with publications. 


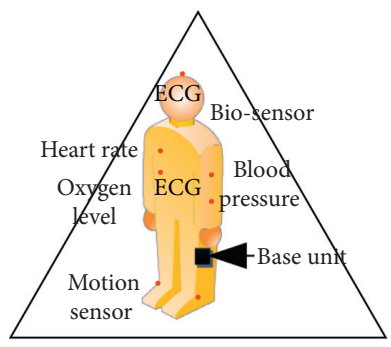

Wireless Body Area Network Tier 1

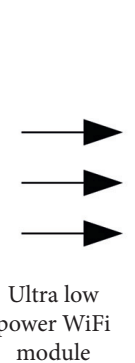

module

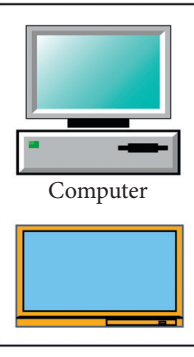

Gateway devices Tier 2
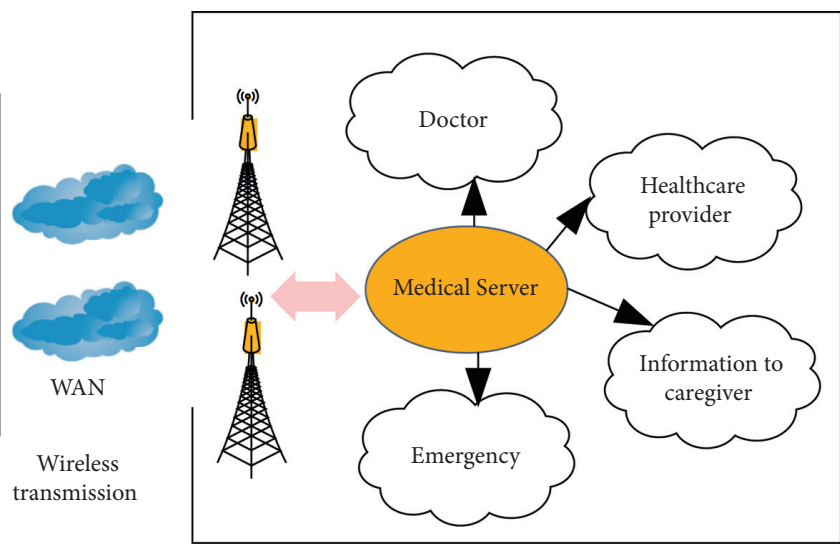

End-user Healthcare monitoring application Tier 3

Figure 1: IoT-based health monitoring system.

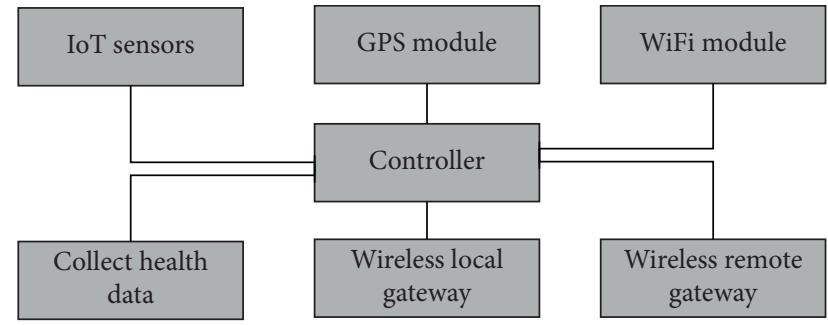

Figure 2: Components involved and architecture.

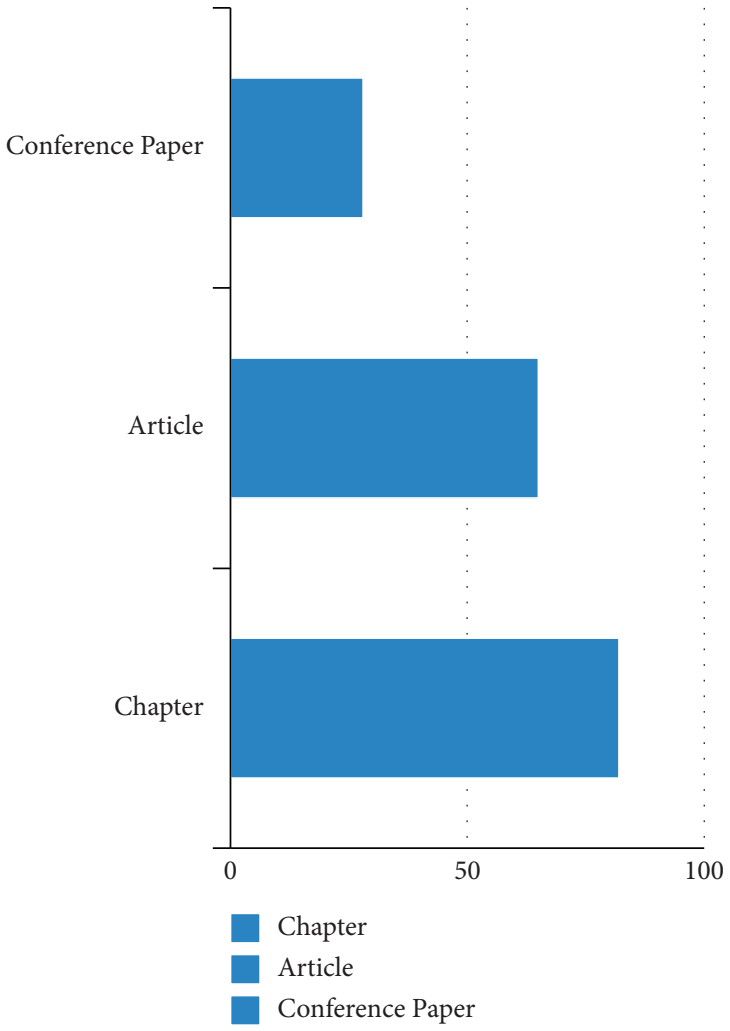

Figure 3: Papers details.

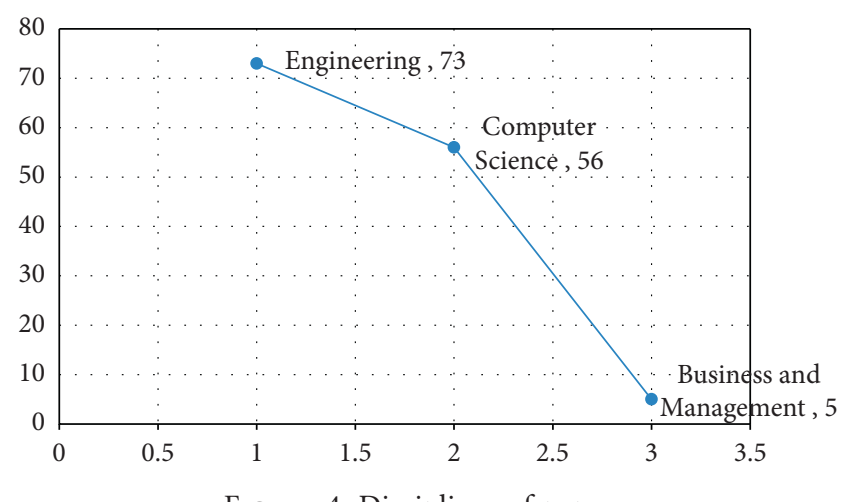

Figure 4: Disciplines of papers.

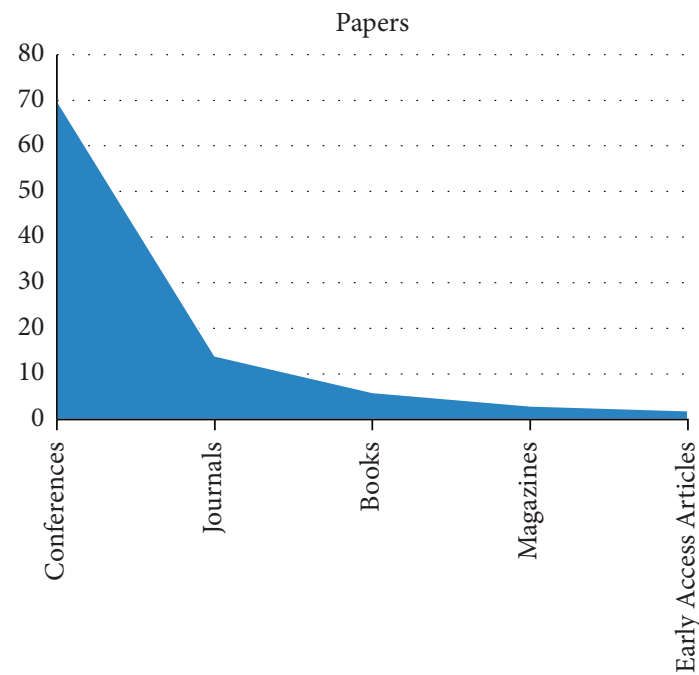

Figure 5: Paper types.

The Wiley online library was considered as search library for identification of relative materials. Figure 13 depicts the papers type with publications.

Figure 14 shows the subjects along with the publications.

Figure 15 is the presentation of papers published in the given library. 


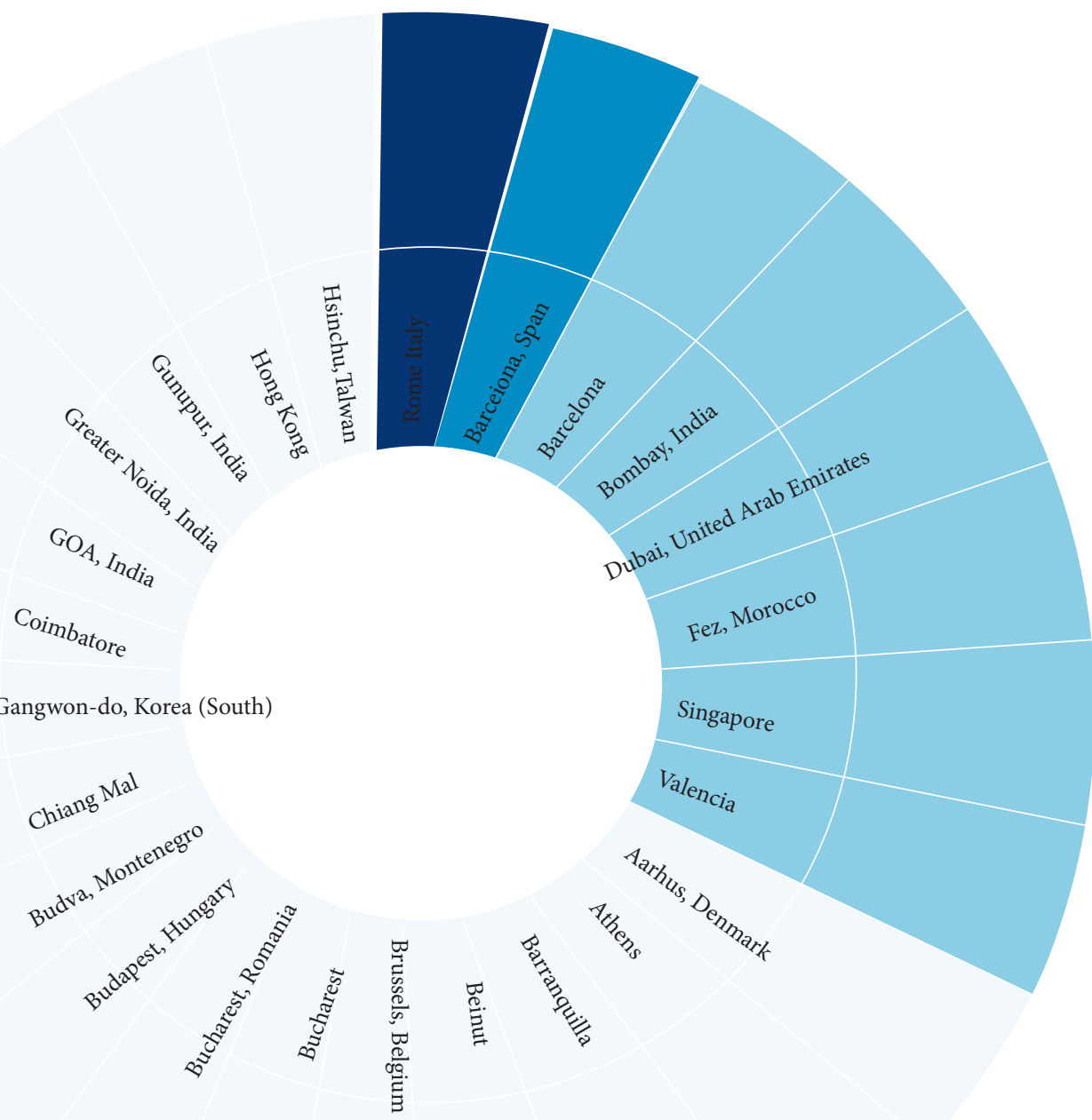

FIgURE 6: Conference locations and papers.

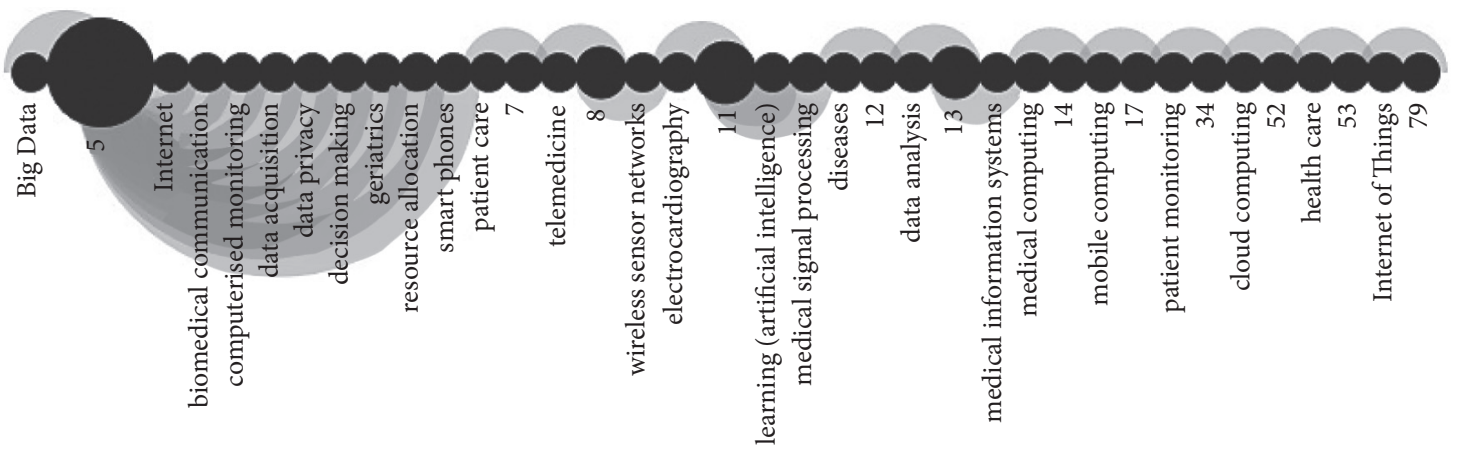

FIgUre 7: Publication topics and papers. 


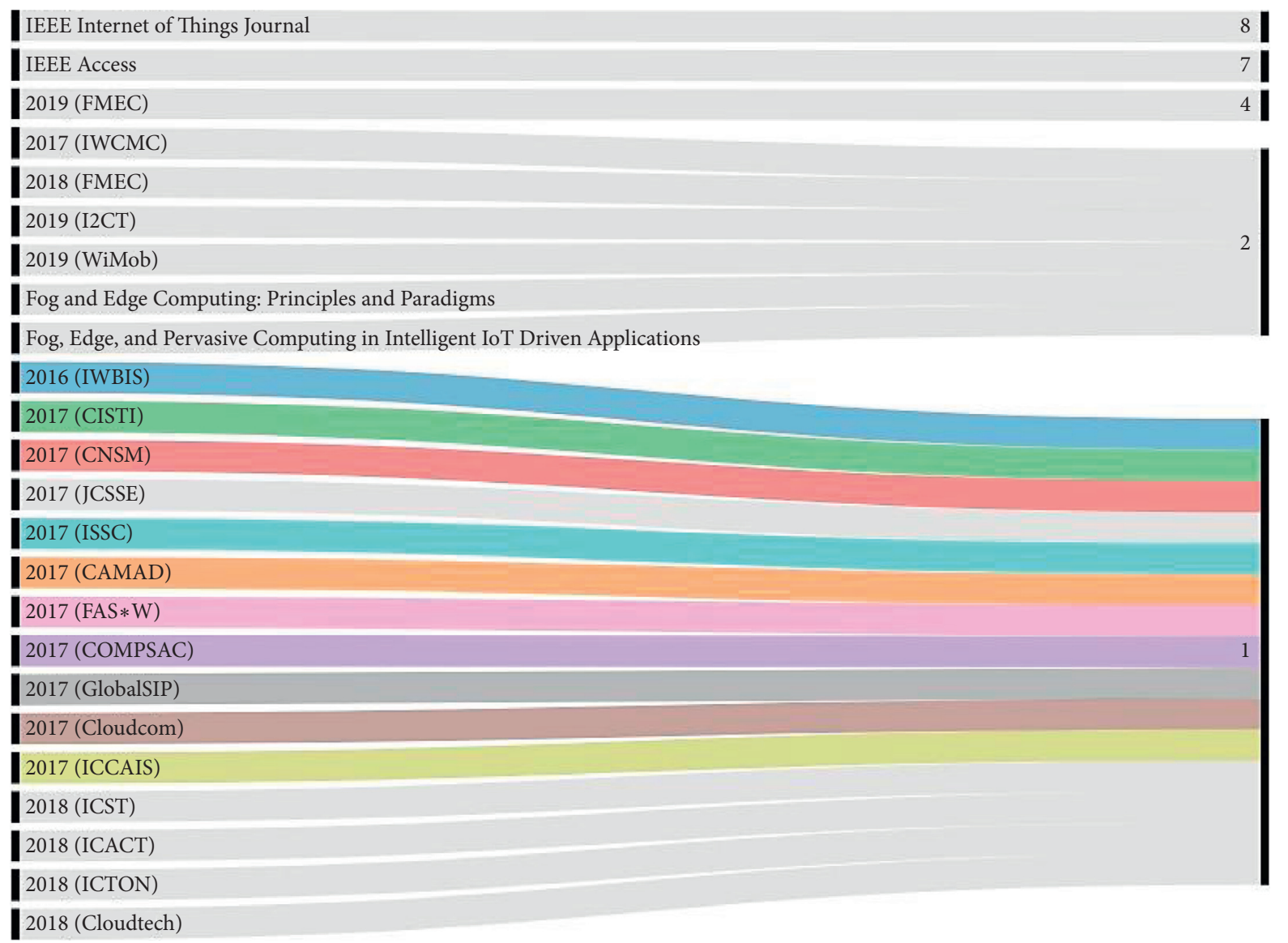

Figure 8: Publication titles and papers.

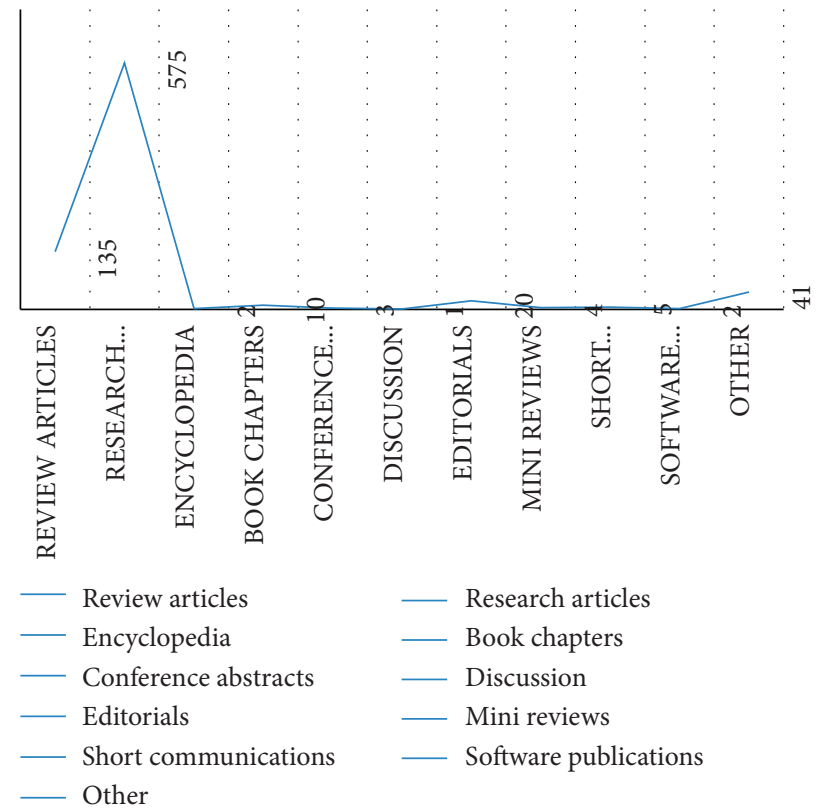

Figure 9: Article type and papers.

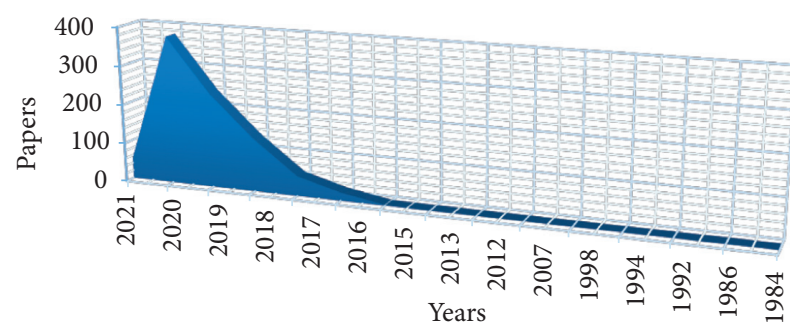

Figure 10: Years and papers.

\section{Selection and Ranking of FC-Based IoT for Monitoring of Health}

Aazam et al. [22] presented a taxonomy of current offloading approaches adopted for the fields such cloud computing, FC, and the IoT. The study has discussed the technologies of middleware enabling offloading in a cloud-IoT case and the significant factors for offloading in a specific scenario. Research prospects associated with offloading in edge and 


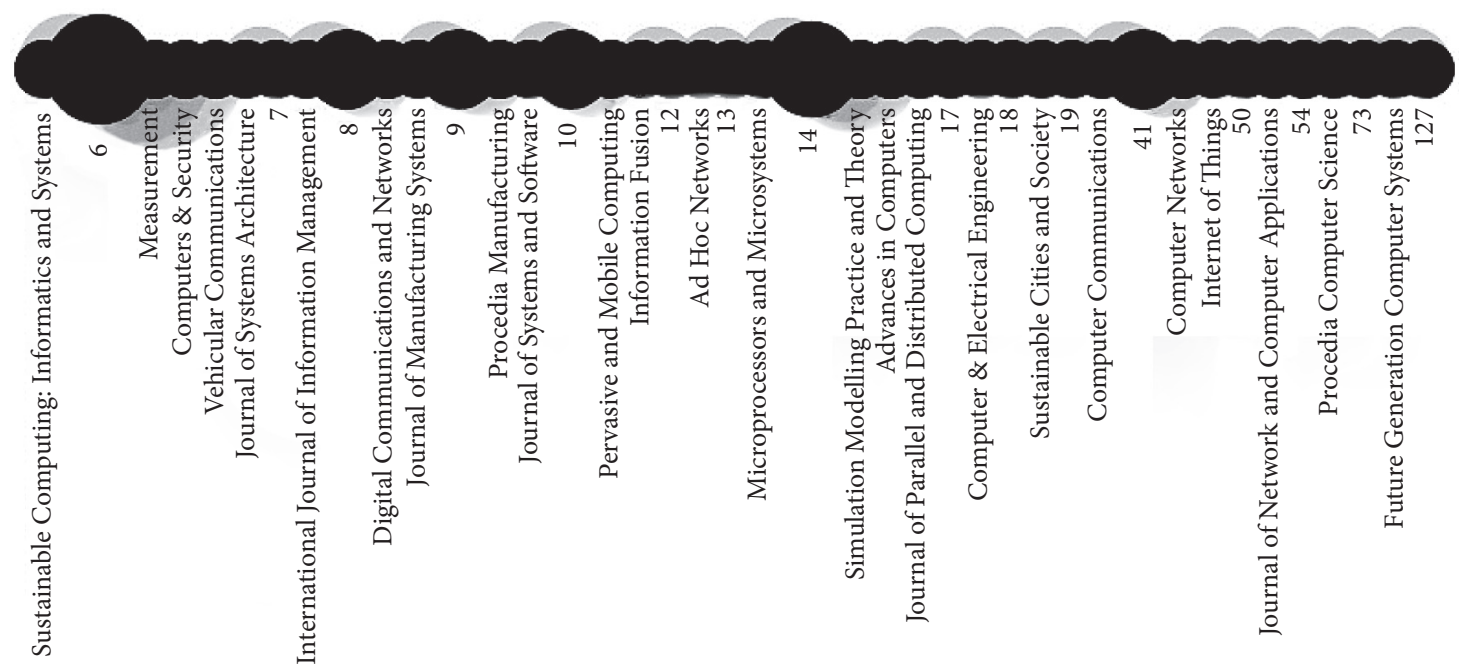

Figure 11: Publication titles and papers.

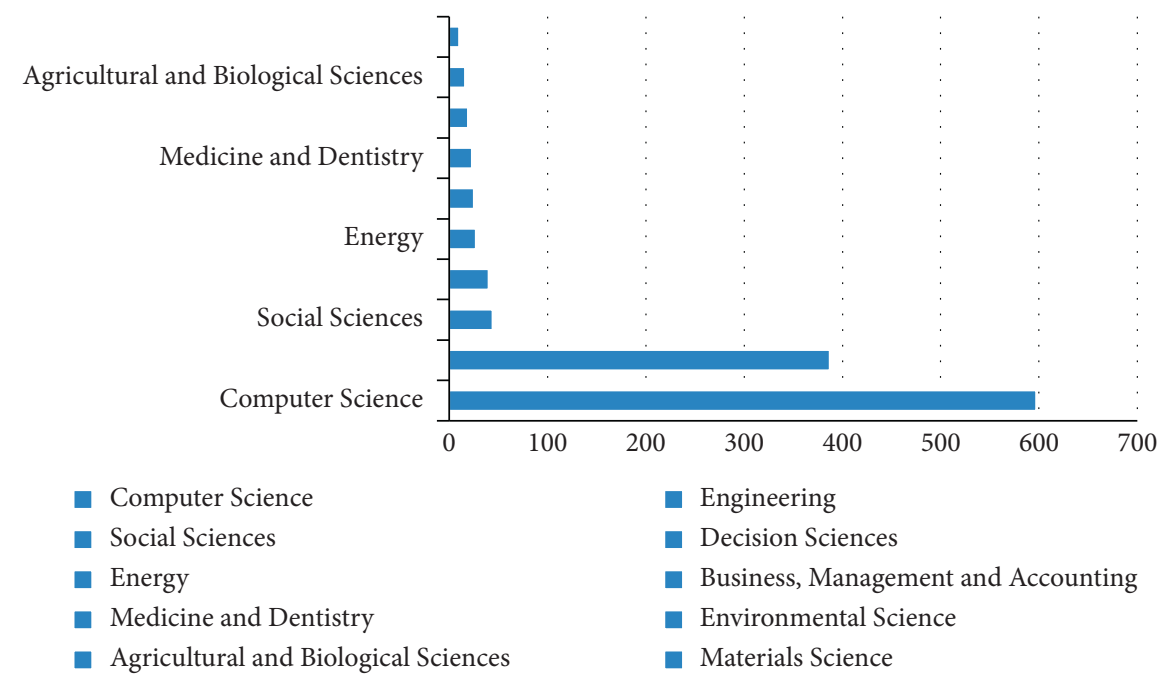

Figure 12: Subject areas and papers.

cloud computing are also presented. Abdelmoneem et al. [23] have proposed an interoperable cloud-fog-based architecture of IoT for healthcare. Diversity of the medical cases and mobility of the patient are supported by the proposed architecture. It demonstrates its architecture, user context, and environmental context. Individual module features are elaborated, and the association between the modules is discussed. Results of the simulations are adequate for cost, latency, and miss ratio. The process of this study was carried out step by step in this section. Table 1 shows the details of the components involved in fog computing IoT (FC-IoT).

The details of the pairwise comparison of Table 1 are shown in Table 2.

The same representation is given for another architecture of the given components. The details are given in Table 3 .

The pairwise comparison of Table 3 is shown in Table 4.

Table 5 shows the architecture for component 1 .
The pairwise comparison process of Table 5 is given in Table 6.

The same process of values is given for architecture 2 and is shown in Table 7.

The pairwise comparison process of Table 7 is shown in Table 8.

The same process was done for rest of the architectures. After completing the process of pairwise comparisons, all the calculated values are brought into a single matrix called weighted matrix. Table 9 represents the weighted matrix.

For taking the decision regarding the components and its architectures, the weighted matrix was then converted into the limit matrix. Table 10 shows the limit matrix.

Figure 16 graphically represents the ranking and selection of FC-based IoT for monitoring of health. The figure shows that FC-IoT 3 got the highest ranking for consideration. 


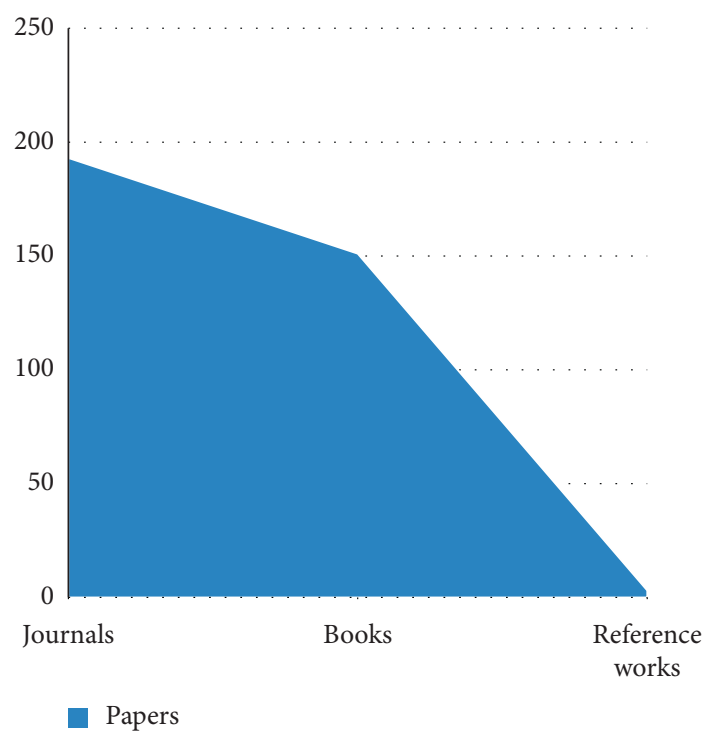

Figure 13: Paper type and papers.

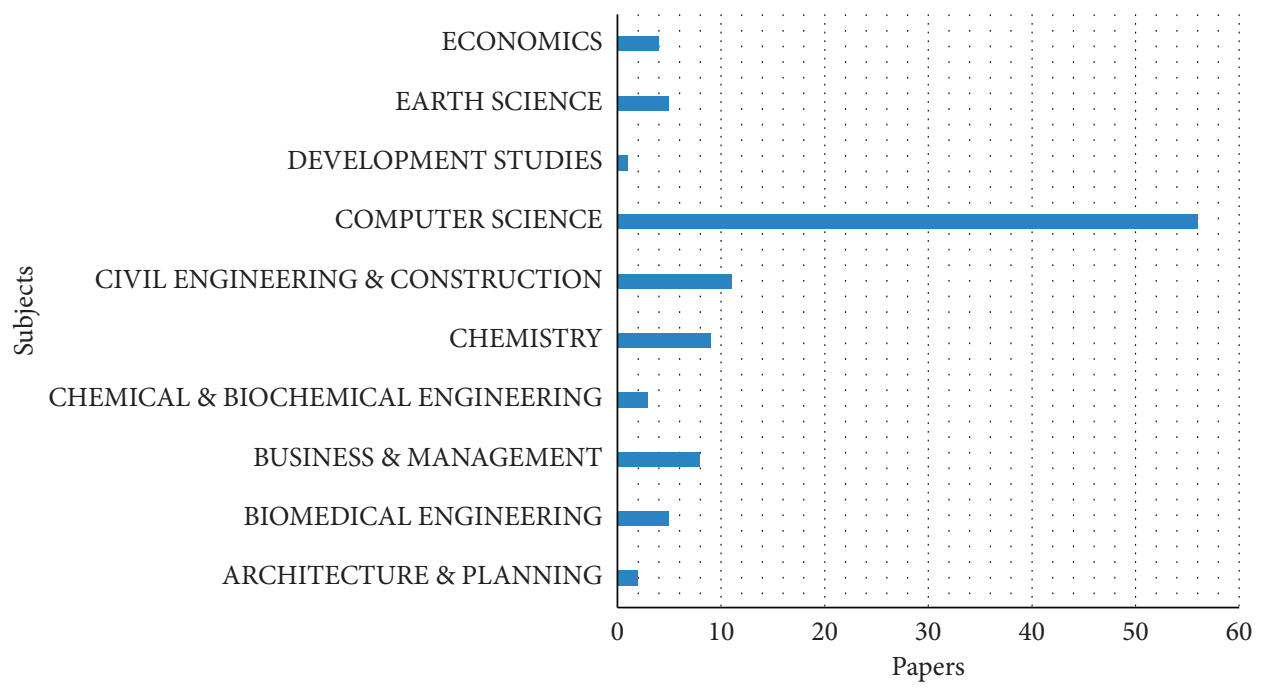

FIgURE 14: Subjects and papers.

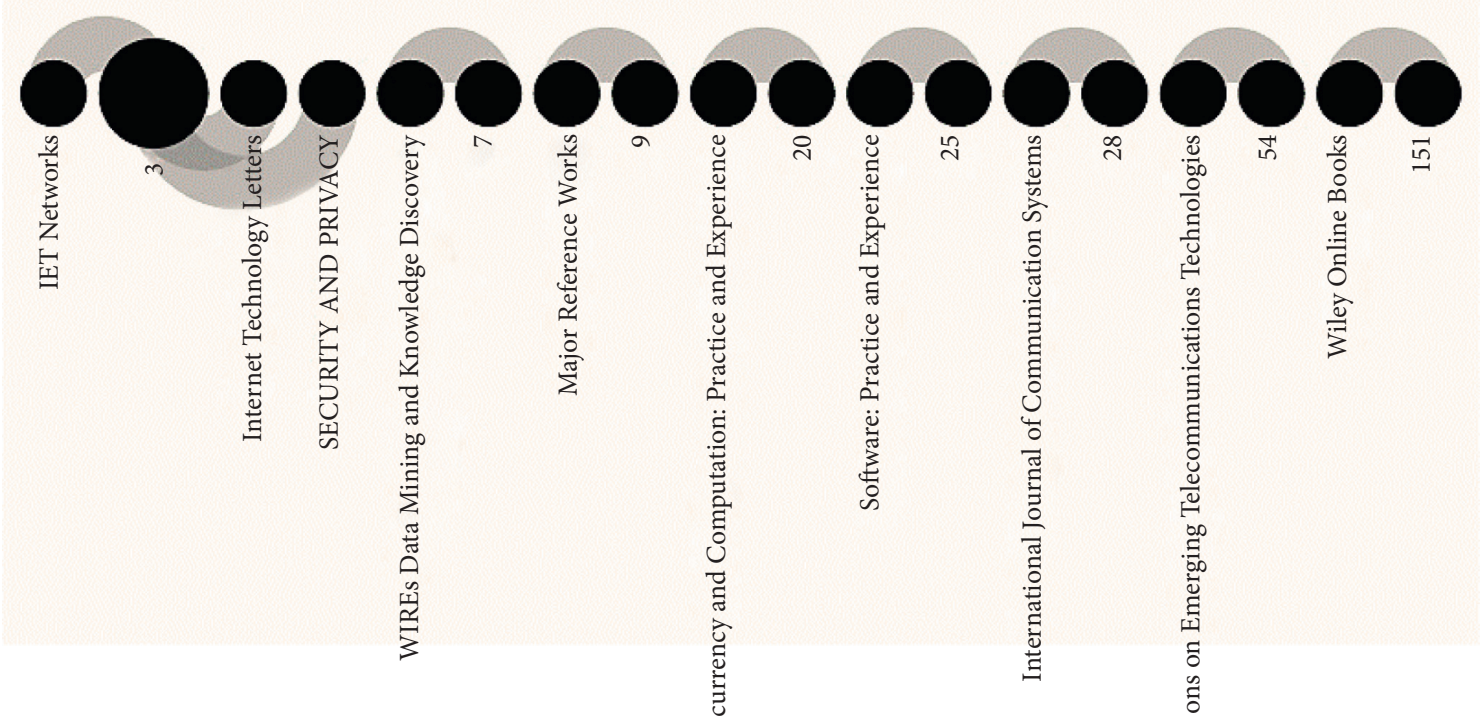

FIGURE 15: Papers published in the given library. 
Table 1: Details of the components involved.

\begin{tabular}{lcccccc}
\hline & IoT sensor & GPS module & WiFi module & $\begin{array}{c}\text { Collect health } \\
\text { data }\end{array}$ & $\begin{array}{c}\text { Wireless local } \\
\text { gateway }\end{array}$ & $\begin{array}{c}\text { Wireless remote } \\
\text { gateway }\end{array}$ \\
\hline IoT sensor & 1 & $1 / 2$ & $1 / 3$ & $1 / 2$ & $1 / 3$ & $1 / 2$ \\
GPS module & 2 & 1 & $1 / 2$ & $1 / 3$ & $1 / 2$ & $1 / 3$ \\
WiFi module & 3 & 2 & 1 & $1 / 3$ & $1 / 2$ & $1 / 2$ \\
Collect health data & 2 & 3 & 3 & 2 & $1 / 2$ & $1 / 2$ \\
Wireless local gateway & 3 & 2 & 3 & 2 & 2 & 1 \\
Wireless remote gateway & 2 & 3 & 2 & & & $1 / 3$ \\
\hline
\end{tabular}

TABle 2: Details of the pairwise comparison.

\begin{tabular}{lcccccc}
\hline & IoT sensor & GPS module & WiFi module & $\begin{array}{c}\text { Collect health } \\
\text { data }\end{array}$ & $\begin{array}{c}\text { Wireless local } \\
\text { gateway }\end{array}$ & $\begin{array}{c}\text { Wireless remote } \\
\text { gateway }\end{array}$ \\
\hline IoT sensor & 0.08 & 0.04 & 0.03 & 0.08 & 0.07 & 0.150 \\
GPS module & 0.15 & 0.09 & 0.05 & 0.05 & 0.11 & 0.100 \\
WiFi module & 0.23 & 0.17 & 0.10 & 0.05 & 0.07 & 0.150 \\
Collect health data & 0.15 & 0.26 & 0.31 & 0.16 & 0.11 & 0.150 \\
Wireless local gateway & 0.23 & 0.17 & 0.31 & 0.32 & 0.32 & 0.43 \\
Wireless remote gateway & 0.15 & 0.26 & 0.20 & 0.300 \\
\hline
\end{tabular}

$\mathrm{CR}=0.083$.

Table 3: Details of components for architecture 2.

\begin{tabular}{lcccccc}
\hline & IoT sensor & GPS module & WiFi module & $\begin{array}{c}\text { Collect health } \\
\text { data }\end{array}$ & $\begin{array}{c}\text { Wireless local } \\
\text { gateway }\end{array}$ & $\begin{array}{c}\text { Wireless remote } \\
\text { gateway }\end{array}$ \\
\hline IoT sensor & 1 & 2 & 3 & 2 & 4 & 3 \\
GPS module & $1 / 2$ & 1 & 3 & 3 & 2 & 3 \\
WiFi module & $1 / 3$ & $1 / 3$ & 1 & 1 & 3 & 2 \\
Collect health data & $1 / 2$ & $1 / 3$ & $1 / 2$ & $1 / 3$ & 1 & 3 \\
Wireless local gateway & $1 / 4$ & $1 / 2$ & $1 / 3$ & $1 / 2$ & $1 / 3$ & 1 \\
Wireless remote gateway & $1 / 3$ & $1 / 3$ & $1 / 2$ & & & 3 \\
\hline
\end{tabular}

TABle 4: Pairwise comparison.

\begin{tabular}{lcccccr}
\hline & $\begin{array}{c}\text { IoT } \\
\text { sensor }\end{array}$ & $\begin{array}{c}\text { GPS } \\
\text { module }\end{array}$ & $\begin{array}{c}\text { WiFi } \\
\text { module }\end{array}$ & $\begin{array}{c}\text { Collect health } \\
\text { data }\end{array}$ & $\begin{array}{c}\text { Wireless local } \\
\text { gateway }\end{array}$ & $\begin{array}{c}\text { Wireless remote } \\
\text { gateway }\end{array}$ \\
\hline IoT sensor & 0.34 & 0.44 & 0.36 & 0.23 & 0.30 & 0.15 \\
GPS module & 0.17 & 0.22 & 0.36 & 0.34 & 0.23 & 0.214 \\
WiFi module & 0.11 & 0.07 & 0.12 & 0.23 & 0.23 & 0.214 \\
Collect health data & 0.17 & 0.07 & 0.06 & 0.11 & 0.243 \\
Wireless local gateway & 0.09 & 0.11 & 0.04 & 0.04 & 0.08 & 0.143 \\
Wireless remote gateway & 0.11 & 0.07 & 0.06 & 0.06 & 0.03 & 0.131 \\
\hline
\end{tabular}

$\mathrm{CR}=0.099$.

TABLe 5: Architecture 1.

\begin{tabular}{lccc}
\hline & FC-IoT 1 & FC-IoT 2 & FC-IoT 3 \\
\hline FC-IoT 1 & 1 & $1 / 2$ & 2 \\
FC-IoT 2 & 2 & 1 & 2 \\
FC-IoT 3 & $1 / 2$ & $1 / 2$ & 1 \\
\hline
\end{tabular}

TABle 6: Pairwise comparison for architecture 1.

\begin{tabular}{lcccc}
\hline & FC-IoT 1 & FC-IoT 2 & FC-IoT 3 & E.V. \\
\hline FC-IoT 1 & 0.29 & 0.25 & 0.40 & 0.31 \\
FC-IoT 2 & 0.57 & 0.50 & 0.40 & 0.49 \\
FC-IoT 3 & 0.14 & 0.25 & 0.20 & 0.20 \\
\hline
\end{tabular}

$\mathrm{CR}=0.052$.
Table 7: Architecture 2.

\begin{tabular}{lccc}
\hline & FC-IoT 1 & FC-IoT 2 & FC-IoT 3 \\
\hline FC-IoT 1 & 1 & 3 & 2 \\
FC-IoT 2 & $1 / 3$ & 1 & 1 \\
FC-IoT 3 & $1 / 2$ & 1 & 1 \\
\hline
\end{tabular}

TABle 8: Pairwise comparison of architecture 2.

\begin{tabular}{lcccc}
\hline & FC-IoT 1 & FC-IoT 2 & FC-IoT 3 & E.V. \\
\hline FC-IoT 1 & 0.55 & 0.60 & 0.50 & 0.55 \\
FC-IoT 2 & 0.18 & 0.20 & 0.25 & 0.21 \\
FC-IoT 3 & 0.27 & 0.20 & 0.25 & 0.24 \\
\hline CR $=0.019$ & & & &
\end{tabular}


TABLE 9: Weighted matrix.

\begin{tabular}{|c|c|c|c|c|c|c|c|c|c|}
\hline & $\begin{array}{c}\text { IoT } \\
\text { sensor }\end{array}$ & $\begin{array}{c}\text { GPS } \\
\text { module }\end{array}$ & $\begin{array}{c}\text { WiFi } \\
\text { module }\end{array}$ & $\begin{array}{c}\text { Collect } \\
\text { health data }\end{array}$ & $\begin{array}{c}\text { Wireless local } \\
\text { gateway }\end{array}$ & $\begin{array}{l}\text { Wireless } \\
\text { remote } \\
\text { gateway }\end{array}$ & FC-IoT 1 & FC-IoT 2 & FC-IoT 3 \\
\hline IoT sensor & 0.000 & 0.000 & 0.000 & 0.000 & 0.000 & 0.000 & 0.076 & 0.315 & 0.338 \\
\hline GPS module & 0.000 & 0.000 & 0.000 & 0.000 & 0.000 & 0.000 & 0.092 & 0.243 & 0.212 \\
\hline WiFi module & 0.000 & 0.000 & 0.000 & 0.000 & 0.000 & 0.000 & 0.130 & 0.150 & 0.158 \\
\hline Collect health data & 0.000 & 0.000 & 0.000 & 0.000 & 0.000 & 0.000 & 0.190 & 0.131 & 0.124 \\
\hline Wireless local gateway & 0.000 & 0.000 & 0.000 & 0.000 & 0.000 & 0.000 & 0.233 & 0.094 & 0.088 \\
\hline $\begin{array}{l}\text { Wireless remote } \\
\text { gateway }\end{array}$ & 0.000 & 0.000 & 0.000 & 0.000 & 0.000 & 0.000 & 0.279 & 0.067 & 0.079 \\
\hline FC-IoT 1 & 0.312 & 0.548 & 0.142 & 0.164 & 0.110 & 0.159 & 0.000 & 0.000 & 0.000 \\
\hline FC-IoT 2 & 0.490 & 0.211 & 0.334 & 0.297 & 0.309 & 0.252 & 0.000 & 0.000 & 0.000 \\
\hline FC-IoT 3 & 0.198 & 0.241 & 0.525 & 0.539 & 0.581 & 0.589 & 0.000 & 0.000 & 0.000 \\
\hline
\end{tabular}

TABle 10: Limit matrix.

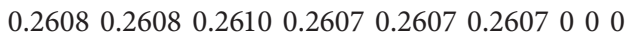

0.19040 .19040 .19050 .19030 .19030 .1903000

$\begin{array}{llllllllllllllll}0.1478 & 0.1478 & 0.1479 & 0.1478 & 0.1478 & 0.1478 & 0 & 0 & 0\end{array}$

$\begin{array}{llllllllllllllllll}0.1437 & 0.1437 & 0.1438 & 0.1437 & 0.1437 & 0.1437 & 0 & 0 & 0\end{array}$

$\begin{array}{llllllllllllllllllllll}0.1283 & 0.1283 & 0.1284 & 0.1283 & 0.1283 & 0.1283 & 0 & 0 & 0\end{array}$

$\begin{array}{llllllllllllllll}0.1279 & 0.1279 & 0.1280 & 0.1279 & 0.1279 & 0.1279 & 0 & 0 & 0\end{array}$

$\begin{array}{lllllllll}0 & 0 & 0 & 0 & 0 & 0 & 0.2648 & 0.2648 & 0.2645\end{array}$

$\begin{array}{llllllllllll}0 & 0 & 0 & 0 & 0 & 0 & 0.3320 & 0.3320 & 0.3317\end{array}$

$\begin{array}{lllllllll}0 & 0 & 0 & 0 & 0 & 0 & 0.4025 & 0.4027 & 0.4022\end{array}$

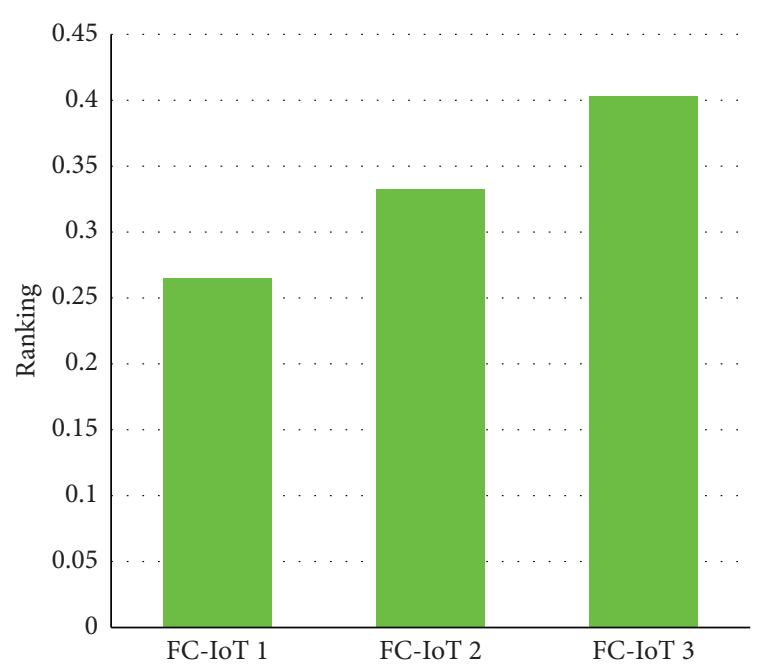

FIGURE 16: Ranking and selection of FC-based IoT for monitoring of health.

\section{Conclusion}

FC is an architecture situated anywhere amongst the cloud and data source where information is computed and stored and the applications are placed to operate the requirements for some specific purpose. Fog computing and edge computing are used by various individuals as both involve understanding and computation together to where the formation of information is done. Numerous platforms and frameworks are considered and proven in the field of FC, applied for various purposes, and are assessed for running analytics on innumerable devices comprising IoT devices and many others in a disseminated way. FC enhances processing, decision, and intervention to take place by devices of the IoT and distributes essential details. The clouds suggested systems improved to overcome several challenges in ubiquitous medical services frameworks, such as manageability, energy efficiency, adaptability, and quality issues through accommodating right to take care of certain weights of the sensor network and a distant medical services group. The research work presented in this paper has used the analytic network process for selection and ranking of FCbased IoT for monitoring of health systems. The experimental results have shown that the proposed research work is efficient in the selection and ranking of fog computing-based IoT for monitoring of health systems. The approach works well in situation when complexity arises for health monitoring.

\section{Data Availability}

No data were used to support this study.

\section{Conflicts of Interest}

The authors declare that they have no conflicts of interest.

\section{References}

[1] A. Paul, H. Pinjari, W.-H. Hong, H. C. Seo, and S. Rho, "Fog computing-based IoT for health monitoring system," Journal of Sensors, vol. 2018, no. 7, Article ID 1386470, 2018.

[2] S. Li, B. Zhang, P. Fei, P. M. Shakeel, R. D. J. Samuel, and V. Behavior, "Computational efficient wearable sensor network health monitoring system for sports athletics using IoT," Aggression and Violent Behavior, Article ID 101541, 2020.

[3] W. Huifeng, S. N. Kadry, and E. D. Raj, "Continuous health monitoring of sportsperson using IoT devices based wearable technology," Computer Communications, vol. 160, pp. 588595, 2020.

[4] A. Ghosh, A. Raha, and A. Mukherjee, "Energy-efficient IoThealth monitoring system using approximate computing," Internet of Things, vol. 9, Article ID 100166, 2020.

[5] S. Juyal, S. Sharma, and A. S. Shukla, "Smart skin health monitoring using AI-enabled cloud-based IoT," Materials Today: Proceedings, 2021.

[6] S. Dhingra, R. B. Madda, R. Patan, P. Jiao, K. Barri, and A. H. Alavi, "Internet of things-based fog and cloud computing technology for smart traffic monitoring," Internet of Things, vol. 4, Article ID 100175, 2020. 
[7] S. K. Sood and I. Mahajan, "Wearable IoT sensor based healthcare system for identifying and controlling chikungunya virus," Computers in Industry, vol. 91, pp. 33-44, 2017.

[8] S. K. Sood and I. Mahajan, "Fog-cloud based cyber-physical system for distinguishing, detecting and preventing mosquito borne diseases," Future Generation Computer Systems, vol. 88, pp. 764-775, 2018.

[9] K. E. Srinivasa Desikan, V. J. Kotagi, and C. Siva Ram Murthy, "Topology control in fog computing enabled IoT networks for smart cities," Computer Networks, vol. 176, Article ID 107270, 2020.

[10] K. Tiwari, S. Kumar, and R. K. Tiwari, "FOG assisted healthcare architecture for pre-operative support to reduce latency," Procedia Computer Science, vol. 167, pp. 1312-1324, 2020.

[11] P. H. Vilela, J. J. P. C. Rodrigues, P. Solic, K. Saleem, and V. Furtado, "Performance evaluation of a fog-assisted IoT solution for e-health applications," Future Generation Computer Systems, vol. 97, pp. 379-386, 2019.

[12] P. Verma, S. K. Sood, and H. Kaur, "A fog-cloud based cyber physical system for ulcerative colitis diagnosis and stage classification and management," Microprocessors and Microsystems, vol. 72, Article ID 102929, 2020.

[13] M. Haghi Kashani, A. M. Rahmani, and N. Jafari Navimipour, "Quality of service-aware approaches in fog computing," International Journal of Communication Systems, vol. 33, no. 8, p. e4340, 2020.

[14] A. Kallel, M. Rekik, and M. Khemakhem, "IoT-fog-cloud based architecture for smart systems: prototypes of autism and COVID-19 monitoring systems," Software: Practice and Experience, vol. 51, no. 1, pp. 91-116, 2021.

[15] G. Suseendran and D. Balaganesh, "Smart cattle health monitoring system using IoT sensors," Materials Today: Proceedings, 2021.

[16] F. Karatas and I. Korpeoglu, "Fog-based data distribution service (F-DAD) for internet of things (IoT) applications," Future Generation Computer Systems, vol. 93, pp. 156-169, 2019.

[17] E. Alemneh, S.-M. Senouci, P. Brunet, and T. Tegegne, "A twoway trust management system for fog computing," Future Generation Computer Systems, vol. 106, pp. 206-220, 2020.

[18] M. Aslam, B. Mohsin, A. Nasir, and S. Raza, "FoNAC-an automated fog node audit and certification scheme," Computers \& Security, vol. 93, Article ID 101759, 2020.

[19] S. Bandopadhaya, R. Dey, and A. Suhag, "Integrated healthcare monitoring solutions for soldier using the internet of things with distributed computing," Sustainable Computing: Informatics and Systems, vol. 26, Article ID 100378, 2020.

[20] R. Bharathi, T. Abirami, S. Dhanasekaran et al., "Energy efficient clustering with disease diagnosis model for IoT based sustainable healthcare systems," Sustainable Computing: Informatics and Systems, vol. 28, Article ID 100453, 2020.

[21] O. Debauche, S. Mahmoudi, P. Manneback, and A. Assila, "Fog IoT for health: a new architecture for patients and elderly monitoring," Procedia Computer Science, vol. 160, pp. 289297, 2019.

[22] M. Aazam, S. Zeadally, and K. A. Harras, "Offloading in fog computing for IoT: review, enabling technologies, and research opportunities," Future Generation Computer Systems, vol. 87, pp. 278-289, 2018.

[23] R. M. Abdelmoneem, A. Benslimane, E. Shaaban, S. Abdelhamid, and S. Ghoneim, "A cloud-fog based architecture for IoT applications dedicated to healthcare," in Proceedings of the ICC 2019-2019 IEEE International Conference on Communications (ICC), pp. 1-6, Shanghai, China, May 2019. 